\title{
Lattice points in bodies with algebraic boundary
}

\author{
by \\ Wolfgang Müller (Graz)
}

1. Introduction. Let $F$ be a polynomial of even degree $d$ in $s$ variables with integer coefficients. Assume that the leading homogeneous part $F^{(d)}$ in the decomposition $F=F^{(d)}+G$ with $\operatorname{deg}(G)<d$ is positive definite. Then $D_{F}(R)=\left\{x \in \mathbb{R}^{d} \mid F(x) \leq R\right\}$ is compact. Denote by $A_{F}(R)$ the number of lattice points of the standard lattice $\mathbb{Z}^{s}$ which are contained in $D_{F}(R)$. Then $A_{F}(R)$ is approximately equal to $\operatorname{vol}\left(D_{F}(R)\right)$. It is easy to see that the discrepancy $P_{F}(R)=A_{F}(R)-\operatorname{vol}\left(D_{F}(R)\right)$ satisfies

$$
P_{F}(R)=\Omega\left(R^{s / d-1}\right) .
$$

One only has to observe that $A_{F}(R+\varepsilon)=A_{F}(R)$ for $R \in \mathbb{N}$ and $0<\varepsilon<1$, but $\operatorname{vol}\left(D_{F}(R+\varepsilon)\right)-\operatorname{vol}\left(D_{F}(R)\right) \gg R^{s / d-1}$. Our aim is to give a sharp upper bound for $P_{F}(R)$. To formulate the main result we introduce the invariant $h(F)$ of $F$, defined as the smallest integer $h$ such that $F^{(d)}$ has a representation

$$
F^{(d)}=\sum_{i=1}^{h} A_{i} B_{i}
$$

with homogeneous polynomials $A_{i}, B_{i} \in \mathbb{Q}\left[X_{1}, \ldots, X_{s}\right]$ of positive degree.

Theorem 1. Assume that $h(F)>\varrho(d)$ where $\varrho(2)=4, \varrho(4)=288$ and $\varrho(d)=d(d-1) 2^{d-1}(\log 2)^{-d} d !$ for $d>4$. Then for $R \geq 1$,

$$
P_{F}(R)=O\left(R^{s / d-1}\right) \text {. }
$$

In the case $d=2$ it is easy to see that $h(F)=s$. Thus Theorem 1 contains as a special case the well known theorem of Walfisz [10] and Landau [4] who proved (2) for rational quadratic forms of dimension $s>4$. If $F^{(d)}$ is non-singular, i.e. the only solution of $\frac{\partial}{\partial x_{i}}\left(F^{(d)}(x)\right)=0,1 \leq i \leq s$, in $\mathbb{C}^{s}$ is $x=0$, then $h(F) \geq s / 2$ (cf. [7, p. 282]). In this case the theorem gives the exact order of $P_{F}(R)$ if $s>2 \varrho(d)$. The proof of Theorem 1 uses a variant of the Hardy-Littlewood method. For general $F$ this method was first used by

2000 Mathematics Subject Classification: 11P21, 11P55.

Key words and phrases: lattice points, Hardy-Littlewood method. 
Schmidt in his famous work on diophantine equations [6], [8]. For special $F$ the estimate (2) can be true for much smaller $s$. As an example we prove

Theorem 2. Let $F_{0}(X)=\sum_{i=1}^{s} \lambda_{i} X_{i}^{d}$ with $d \geq 2$ even and integer coefficients $\lambda_{i}>0$. Then $P_{F_{0}}(R)=O\left(R^{s / d-1}\right)$, provided that $s \geq \min \left(d 2^{d-1}\right.$, $\left.\varrho_{0}(d)\right)$. Here $\varrho_{0}$ denotes an explicitly computable function which satisfies $\varrho_{0}(d) \sim 2 d^{3} \log d$ for $d \rightarrow \infty$.

As noted by Randol [5] Theorem 2 cannot be true if $s<d^{2}-d+1$. See Krätzel [3] for a detailed study of $P_{F_{0}}(R)$ for small $s$. With some obvious modifications our proof shows that Theorem 2 remains true for real coefficients $\lambda_{i}>0$.

Recently, Bentkus and Götze [1] studied $P_{F}(R)$ for polynomials $F$ with real coefficients and leading homogeneous part

$$
F^{(d)}(X)=\sum_{i=1}^{s_{0}} \lambda_{i} X_{i}^{d}+P(X) \quad\left(\lambda_{i}>0\right) .
$$

Here $P$ denotes a homogeneous polynomial of degree $d$ such that the degree of $P$ viewed as a polynomial in $\left(X_{1}, \ldots, X_{s_{0}}\right)$ is strictly smaller than $d$. They proved (2) under the assumptions that $s_{0}=s$ and $s>\alpha(d)$ or $s_{0}<s$ and $s_{0}>2^{d} \alpha(d)$, where $\alpha(2)=8, \alpha(4)=1512$ and $\alpha(d)=d 2^{d-1} e^{3 d \log d}$ for $d>4$. The condition (3) on the leading homogeneous part of $F$ is rather restrictive. Bentkus and Götze already remarked that one should expect that (2) is true for general $F$ if $h(F)$ is sufficiently large. The main advantage of their method is that it applies to polynomials with real coefficients, whereas we have to assume that $F$ has integer coefficients.

2. The Hardy-Littlewood method. Let $B=(-1,1]^{\text {s }}$. Assume that $R \in \mathbb{N}$ and $D_{F}(R) \subseteq R^{1 / d} B$ for $R \geq c(F)$ sufficiently large. Otherwise consider $c F$ instead of $F$, where $c \in \mathbb{N}$ is sufficiently large, and use $A_{F}(R)=$ $A_{c F}(c R)$. To count the number of lattice points in $D_{F}(R)$ we introduce the auxiliary function $\chi=I_{(-R-1 / 2, R+1 / 2)} * \delta$ which is the convolution of the indicator function with a symmetric probability density $\delta \in \mathbb{C}^{\infty}(\mathbb{R})$ satisfying $\operatorname{supp}(\delta) \subseteq[-1 / 2,1 / 2]$. Then $\chi(u)=1$ if $|u| \leq R, \chi(u)=0$ if $|u| \geq R+1$ and $0 \leq \chi(u) \leq 1$ if $R<|u|<R+1$. By Fourier inversion one obtains

$$
\chi(u)=\int_{\mathbb{R}} \widehat{\chi}(t) e(-t u) d t=\int_{\mathbb{R}} \widehat{\chi}(t) e(t u) d t
$$

where

$$
\widehat{\chi}(t)=\int_{\mathbb{R}} \chi(u) e(t u) d u=\widehat{I}_{(-R-1 / 2, R+1 / 2)}(t) \widehat{\delta}(t) .
$$


Here $e(x)=e^{2 \pi i x}$ as usual. Furthermore,

$$
\widehat{I}_{(-R-1 / 2, R+1 / 2)}(t)=\frac{1}{\pi t} \sin (2 \pi t(R+1 / 2)) .
$$

Applying $j$-fold partial integration one obtains $\widehat{\delta}(t) \ll_{j}(|t|+1)^{-j}$ for $j \geq 0$. Hence

$$
\widehat{\chi}(t) \ll \frac{1}{|t|}(1+|t|)^{-j} \quad(j \geq 0) .
$$

Set $N=\left\lceil(R+1)^{1 / d}\right\rceil+1 / 2$. Then $F(k) \leq R$ implies $k \in N B$ and (4) yields

with

$$
A_{F}(R)=\sum_{n \in N B \cap \mathbb{Z}^{s}} \chi(F(n))=\int_{\mathbb{R}} S_{N}(t) \widehat{\chi}(t) d t
$$

$$
S_{N}(t)=\sum_{n \in N B \cap \mathbb{Z}^{s}} e(t F(n)) .
$$

This should be compared with the following integral which counts the number of lattice points on the boundary of $D_{F}(R)$ :

$$
\int_{0}^{1} S_{N}(t) e(-t R) d t .
$$

It is not surprising that the properties of $S_{N}(t)$ known from the HardyLittlewood method can be used to analyse $A_{F}(R)$. The main difference comes from the behaviour of $\widehat{\chi}(t)$ for small $t$. Note that $S_{N}(t)$ is one-periodic if $F$ has integer coefficients. The following proposition deals with these small values of $t$.

Proposition. Assume that for $N \geq 1$ :

(A) $\int\left|S_{N}(t)\right| d t \ll N^{s-d}$. $(0,1]$

(B) $\int_{\left(N^{1-d}, 1\right]}\left|S_{N}(t)\right| \frac{d t}{t} \ll N^{s-d}$.

(C) There exists an $\omega>d$ such that for $|t| \leq N^{1-d}$

$$
\sum_{n \in N B^{\prime} \cap \mathbb{Z}^{s}} e(t F(n+u)) \ll N^{s-\omega d}|t|^{-\omega}
$$

uniformly in $u \in B$ and all boxes $B^{\prime} \subseteq B$ with sides parallel to the coordinate axes.

(D) There exists an $\omega>d$ such that for $|t| \geq N^{-d}$,

$$
\int_{N B^{\prime}} e(t F(x)) d x \ll N^{s-\omega d}|t|^{-\omega}
$$

uniformly in all boxes $B^{\prime} \subseteq B$ with sides parallel to the coordinate axes.

Then $P_{F}(R) \ll R^{s / d-1}$. 
The proof of this Proposition is given in Section 3. Here we describe the "axiomatic" form of the Hardy-Littlewood method given by Schmidt [6]. If $F$ is a polynomial with integer coefficients, $S_{N}(t)$ can be evaluated asymptotically in a neighbourhood of a rational number with small denominator. The union of these neighbourhoods is called the major arcs. To be precise let $0<\Delta \leq 1$ and set, for $1 \leq a \leq q \leq N^{\Delta}$ with $(a, q)=1$,

$$
\mathfrak{M}_{\Delta}(q, a)=\left\{t \in \mathbb{R} / \mathbb{Z}|| t-\frac{a}{q} \mid<\frac{1}{q} N^{\Delta-d}\right\} .
$$

Then the major arcs and minor arcs are defined by

$$
\mathfrak{M}_{\Delta}=\bigcup_{\substack{1 \leq a \leq q \leq N^{\Delta} \\(a, q)=1}} \mathfrak{M}_{\Delta}(q, a) \quad \text { and } \quad \mathfrak{m}_{\Delta}=(\mathbb{R} / \mathbb{Z}) \backslash \mathfrak{M}_{\Delta}
$$

Note that $\mathfrak{M}_{\Delta}$ is the union of disjoint intervals if $N$ is sufficiently large.

If $F$ is homogeneous, i.e. $F=F^{(d)}$, we define $\Omega(F)$ as the supremum of all $\omega>0$ such that for all $\Delta \in(0,1]$ and $t \in \mathfrak{m}_{\Delta}$,

$$
\sum_{n \in N B^{\prime} \cap \mathbb{Z}^{s}} e(t F(n+u)) \ll_{F, \omega} N^{s-\omega \Delta}
$$

uniformly for all $u \in B$ and all boxes $B^{\prime} \subseteq B$ with sides parallel to the coordinate axes. If $F$ is an arbitrary polynomial with leading form $F^{(d)}$ we define $\Omega(F)$ as the supremum of all $\omega>0$ such that for all $\Delta \in(0,1]$ and $t \in \mathfrak{m}_{\Delta}$,

$$
\sum_{n \in N B^{\prime} \cap \mathbb{Z}^{s}} e\left(t F^{(d)}(n)+P(n)\right) \ll_{F, \omega} N^{s-\omega \Delta}
$$

uniformly for all polynomials $P \in \mathbb{R}\left[X_{1}, \ldots, X_{s}\right]$ with $\operatorname{deg}(P)<d$ and all boxes $B^{\prime} \subseteq B$ with sides parallel to the coordinate axes.

$\Omega(F)$ is similar to the invariant $\omega(F)$ introduced by Schmidt [6]. The latter is defined as the supremum of all $\omega>0$ such that for all $\Delta \in(0,1]$ and $t \in \mathfrak{m}_{\Delta},(9)$ is true with $u=0$ uniformly for all boxes $B^{\prime} \subseteq B$. We prove that the assumption $\Omega(F)>d$ implies (A)-(D) of the above Proposition.

Theorem 3. If $\Omega(F)>d$ then $P_{F}(R) \ll R^{s / d-1}$.

Theorem 1 follows immediately from Theorem 3 and the following inequality:

$$
\Omega(F) \geq \frac{h(F)}{\tau(d)} .
$$

Here $\tau(2)=2, \tau(4)=72$ and $\tau(d)<(d-1) 2^{d-1}(\log 2)^{-d} d$ ! in general. With $\Omega(F)$ replaced by $\omega(F)$ this is Theorem 6.A in [6, p. 86]. We have to verify that Schmidt's inequality remains true with our modified invariant $\Omega(F)$. To see this note that Schmidt's proof starts with a $d$-fold application 
of Weyl's inequality. This transforms the exponential sum in the definition of $\Omega(F)$ into an exponential sum of the form $\sum e\left(G_{d}\left(n_{1}, \ldots, n_{d}\right)\right)$, where $G(X)=t F^{(d)}(X)+P(X)$ and $G_{d}$ is the unique symmetric multilinear form which satisfies $G^{(d)}(X)=\frac{(-1)^{d}}{d !} G_{d}(X, \ldots, X)$. If $P$ is a polynomial of degree strictly less than $d$, then $P_{d}=0$. It follows that $G_{d}=t F_{d}^{(d)}$. Hence the new exponential sum does not depend on $P$. From this moment on, one proceeds as in [6]. Note that $\Omega(F)$ and the above lower bound on $\Omega(F)$ depend only on the leading form of $F$.

3. Proof of the Proposition. Assume that conditions (A)-(D) of the Proposition are satisfied. The representation (6), together with (5), (A) and $(\mathrm{B})$, yields

$$
\begin{aligned}
A_{F}(R)= & \int_{|t| \leq N^{1-d}} S_{N}(t) \widehat{\chi}(t) d t \\
& +O\left(\int_{\left(N^{1-d}, 1\right]}\left|S_{N}(t)\right| \frac{d t}{t}+\sum_{j=1}^{\infty} \frac{1}{j^{2}} \int_{(j, j+1]}\left|S_{N}(t)\right| d t\right) \\
= & \int_{|t| \leq N^{1-d}} S_{N}(t) \widehat{\chi}(t) d t+O\left(N^{s-d}\right) .
\end{aligned}
$$

If $|t| \leq N^{1-d}$ we use an asymptotic expansion of $S_{N}(t)$. There are several ways to obtain it. We use the following expansion of a sufficiently smooth complex-valued function $g: \mathbb{R}^{s} \rightarrow \mathbb{C}$ due to Bentkus and Götze [1]. Let $J \in \mathbb{N}$, and $x, u_{1}, \ldots, u_{J} \in \mathbb{R}^{s}$. Then

$$
g(x)=g\left(x+u_{1}\right)+\sum_{j=1}^{J-1} g_{j}+r_{J}
$$

where for $1 \leq j<J$,

$$
g_{j}=\sum_{|\alpha|=j} c(\alpha) g^{(j)}\left(x+u_{m+1}\right)\left[u_{1}^{\alpha_{1}} \ldots u_{m}^{\alpha_{m}}\right]
$$

and

$$
r_{J}=\sum_{|\alpha|=J} c^{\prime}(\alpha) \int_{0}^{1}(1-\tau)^{\alpha_{m}-1} g^{(J)}\left(x+\tau u_{m}\right)\left[u_{1}^{\alpha_{1}} \ldots u_{m}^{\alpha_{m}}\right] d \tau .
$$

The summation extends over all $\alpha=\left(\alpha_{1}, \ldots, \alpha_{m}\right) \in \mathbb{N}^{m}$ with $1 \leq m \leq j$ and $|\alpha|=\sum_{i=1}^{m} \alpha_{i}=j$. Furthermore, $g^{(j)}(x)\left[u_{1}^{\alpha_{1}} \ldots u_{m}^{\alpha_{m}}\right]$ denotes the $j$-fold directional derivative

$$
g^{(j)}(x)\left[u_{1}^{\alpha_{1}} \ldots u_{m}^{\alpha_{m}}\right]=\left.\frac{\partial^{j}}{\partial \lambda_{1}^{\alpha_{1}} \ldots \partial \lambda_{m}^{\alpha_{m}}} g\left(x+\lambda_{1} u_{1}+\ldots+\lambda_{m} u_{m}\right)\right|_{\lambda_{1}=\ldots=\lambda_{m}=0}
$$


and

$$
c(\alpha)=\frac{(-1)^{m}}{\alpha_{1} ! \ldots \alpha_{m} !}, \quad c^{\prime}(\alpha)=\frac{(-1)^{m}}{\alpha_{1} ! \ldots \alpha_{m-1} !\left(\alpha_{m}-1\right) !} .
$$

This expansion can be obtained by iteratively applying Taylor expansions, first to $\lambda \mapsto g\left(x+\lambda u_{1}\right)$ and then for every summand $g^{\left(\alpha_{1}\right)}(x)\left[u_{1}^{\alpha_{1}}\right]$ in the resulting expansion to $\lambda \mapsto g^{\left(\alpha_{1}\right)}\left(x+\lambda u_{2}\right)\left[u_{1}^{\alpha_{1}}\right]$. After $J$ such steps one obtains (13).

We use (13) with $g(x)=e(t F(x))$. Summing over $x \in N B \cap \mathbb{Z}^{s}$ and integrating over $\left(u_{1}, \ldots, u_{J}\right) \in T^{J}$ with $T=(-1 / 2,1 / 2]^{s}$, yields

$$
S_{N}(t)=G_{0}(t)+\sum_{j=1}^{J-1} G_{j}(t)+R_{J}(t),
$$

where

$$
\begin{aligned}
G_{0}(t)= & \int_{T} \sum_{x \in N B \cap \mathbb{Z}^{s}} g\left(x+u_{1}\right) d u_{1}=\int_{N B} g(x) d x \\
G_{j}(t)= & \sum_{|\alpha|=j} c(\alpha) \int_{T^{m}}\left(\int_{N B} g^{(j)}(x)\left[u_{1}^{\alpha_{1}} \ldots u_{m}^{\alpha_{m}}\right] d x\right) d\left(u_{1}, \ldots, u_{m}\right), \\
R_{J}(t)= & \sum_{|\alpha|=J} c^{\prime}(\alpha) \int_{0}^{1}(1-\tau)^{\alpha_{m}-1} \\
& \times \int_{T^{m}} \sum_{x \in N B \cap \mathbb{Z}^{s}} g^{(J)}\left(x+\tau u_{m}\right)\left[u_{1}^{\alpha_{1}} \ldots u_{m}^{\alpha_{m}}\right] d\left(u_{1}, \ldots, u_{m}\right) d \tau .
\end{aligned}
$$

With the choice $J=d$ we prove that

$$
\int_{|t| \leq N^{1-d}} R_{d}(t) \widehat{\chi}(t) d t \ll N^{s-d}
$$

and for $0 \leq j<d$,

$$
\int_{|t|>N^{1-d}} G_{j}(t) \widehat{\chi}(t) d t \ll N^{s-d}
$$

From this it follows that

$$
\begin{aligned}
\int_{|t| \leq N^{1-d}} S_{N}(t) \widehat{\chi}(t) d t & =\sum_{j=0}^{d-1} \int_{|t| \leq N^{1-d}} G_{j}(t) \widehat{\chi}(t) d t+O\left(N^{s-d}\right) \\
& =\sum_{j=0}^{d-1} H_{j}+O\left(N^{s-d}\right)
\end{aligned}
$$

where

$$
H_{j}=\int_{\mathbb{R}} G_{j}(t) \widehat{\chi}(t) d t
$$


Together with (12) and the definition of $N$ we obtain

$$
A_{F}(R)=\sum_{j=0}^{d-1} H_{j}+O\left(R^{s / d-1}\right) .
$$

$H_{0}$ yields the main term since

$$
\begin{aligned}
H_{0} & =\int_{\mathbb{R}} G_{0}(t) \widehat{\chi}(t) d t=\int_{N B \mathbb{R}} \int_{F} e(t F(x)) \widehat{\chi}(t) d t d x=\int_{N B} \chi(F(x)) d x \\
& =\int_{F(x) \leq R} d x+O\left(\int_{R<F(x) \leq R+1} d x\right)=\operatorname{vol}\left(D_{F}(R)\right)+O\left(R^{s / d-1}\right) .
\end{aligned}
$$

In the remaining part of this section we prove (15), (16) and $H_{j}=0$ for $j \geq 1$. This will complete the proof of the Proposition. We begin with the following lemma which can be proved by induction.

Lemma 3.1. Let $g(x)=e(t F(x))$ and $x, u_{1}, \ldots, u_{j} \in \mathbb{R}^{s}$. Then

$$
g^{(j)}(x)\left[u_{1}, \ldots, u_{j}\right]=g(x) \sum_{l=1}^{j}(2 \pi i t)^{l} P_{j, l}(x),
$$

where $P_{j, l}, 1 \leq l \leq j$, are polynomials with $\operatorname{deg}\left(P_{j, l}\right) \leq l d-j$ whose coefficients are linear in $u_{1}, \ldots, u_{j}$. They can be determined recursively by

$$
\begin{aligned}
P_{j+1,1}(x) & =\sum_{i=1}^{s} \frac{\partial}{\partial x_{i}}\left(P_{j, 1}(x)\right) u_{j+1}^{(i)}, \\
P_{j+1, l}(x) & =\sum_{i=1}^{s} \frac{\partial}{\partial x_{i}}\left(P_{j, l}(x)\right) u_{j+1}^{(i)}+P_{j, l-1}(x) \sum_{i=1}^{s} \frac{\partial F}{\partial x_{i}}(x) u_{j+1}^{(i)} \quad(2 \leq l \leq j), \\
P_{j+1, j+1}(x) & =P_{j, j}(x) \sum_{i=1}^{s} \frac{\partial F}{\partial x_{i}}(x) u_{j+1}^{(i)},
\end{aligned}
$$

and

$$
P_{1,1}(x)=\sum_{i=1}^{s} \frac{\partial F}{\partial x_{i}}(x) u_{1}^{(i)} .
$$

Here $u_{j}^{(i)}$ denotes the ith component of $u_{j}$.

To prove (15) we consider the cases $|t| \leq N^{-d}$ and $N^{-d}<|t| \leq N^{1-d}$ separately. If $|t| \leq N^{-d}$ we estimate $g^{(d)}$ trivially. Since $P_{j, l}(x) \ll N^{l d-j}$ uniformly in $u_{1}, \ldots, u_{j} \in T$ and $x \in 2 N B$, (17) and $|t| N^{d} \leq 1$ imply $g^{(j)}(x)\left[u_{1}, \ldots, u_{j}\right] \ll|t| N^{d-j}$. Hence $R_{d}(t) \ll|t| N^{s}$. Together with $\widehat{\chi}(t) \ll$ $|t|^{-1}$ this yields

$$
\int_{|t| \leq N^{-d}} R_{J}(t) \widehat{\chi}(t) d t \ll \int_{|t| \leq N^{-d}} N^{s} d t \ll N^{s-d} .
$$


In the case $N^{-d}<|t| \leq N^{1-d}$ we use assumption (C). Since the estimate in (C) is uniform in all boxes $B^{\prime} \subseteq B$ with sides parallel to the coordinate axes we can apply partial summation. This yields, for an arbitrary polynomial $P$,

$$
\sum_{n \in N B} e(t F(n+u)) P(n+u) \ll N^{\operatorname{deg}(P)+s-\omega d}|t|^{-\omega}
$$

uniformly in $u \in T$. Together with (17) we obtain

$$
\begin{aligned}
\sum_{n \in N B \cap \mathbb{Z}^{s}} g^{(d)}(n+ & \left.\tau u_{m}\right)\left[u_{1}^{\alpha_{1}} \ldots u_{m}^{\alpha_{m}}\right] \\
& =\sum_{l=1}^{d}(2 \pi i t)^{l} \sum_{n \in N B \cap \mathbb{Z}^{s}} P_{d, l}\left(n+\tau u_{m}\right) e\left(t F\left(n+\tau u_{m}\right)\right) \\
& \ll N^{-d+s-\omega d}|t|^{-\omega} \sum_{l=1}^{d}\left(|t| N^{d}\right)^{l} \ll N^{d^{2}-d+s-\omega d}|t|^{d-\omega} .
\end{aligned}
$$

Since $\omega>d$ it follows that

$$
\int_{\left(N^{-d}, N^{1-d}\right]} R_{d}(t) \widehat{\chi}(t) d t \ll N^{d^{2}-d+s-\omega d} \int_{\left(N^{-d}, N^{1-d}\right]} t^{d-\omega-1} d t \ll N^{s-d} .
$$

This together with (18) implies (15).

To prove (16) we use (D). Since the estimate in (D) is uniform in all boxes $B^{\prime} \subseteq B$ we can apply partial integration. This gives, for an arbitrary polynomial $P$ and $|t| \geq N^{-d}$,

$$
\int_{N B} P(x) e(t F(x)) d x \ll N^{\operatorname{deg}(P)+s-\omega d}|t|^{-\omega} .
$$

Hence Lemma 3.1 implies, for $|t| \geq N^{-d}$ (uniformly in $u_{1}, \ldots, u_{m} \in T$ ),

$$
\begin{aligned}
\int_{N B} g^{(j)}(x)\left[u_{1}^{\alpha_{1}} \ldots u_{m}^{\alpha_{m}}\right] d x & =\sum_{l=1}^{j} \int_{N B}(2 \pi i t)^{l} P_{j, l}(x) e(t F(x)) d x \\
& \ll N^{s-j-\omega d}|t|^{-\omega} \sum_{l=1}^{j}\left(|t| N^{d}\right)^{l} \ll N^{s+j(d-1)-\omega d}|t|^{j-\omega} .
\end{aligned}
$$

For $0 \leq j<d$ this together with (5) yields

$$
\int_{|t|>N^{1-d}} G_{j}(t) \widehat{\chi}(t) d t \ll N^{s+j(d-1)-\omega d}\left(\int_{\left(N^{1-d}, 1\right]} t^{j-\omega-1} d t+\int_{(1, \infty)} t^{-2} d t\right) \ll N^{s-\omega} .
$$

Since $\omega>d$ this implies (16).

Finally, we prove

Lemma $3.2 . H_{j}=0$ for $j \geq 1$. 
Proof. By Lemma 3.1 and the definition of $H_{j}$ we obtain, for $j \geq 1$,

$$
\begin{aligned}
H_{j} & =\int_{\mathbb{R}} G_{j}(t) \widehat{\chi}(t) d t \\
& =\sum_{|\alpha|=j} c(\alpha) \iint_{\mathbb{R} T^{m}} \int_{N B} g^{(j)}(x)\left[u_{1}^{\alpha_{1}} \ldots u_{m}^{\alpha_{m}}\right] \widehat{\chi}(t) d x d\left(u_{1} \ldots u_{m}\right) d t \\
& =\sum_{|\alpha|=j} c(\alpha) \int_{T^{m}} \sum_{l=1}^{j} \int_{N B} P_{j, l}(x) \int_{\mathbb{R}} e(t F(x)) \widehat{\chi^{(l)}}(t) d t d x d\left(u_{1} \ldots u_{m}\right) \\
& =\sum_{|\alpha|=j} c(\alpha) \int_{T^{m}} \sum_{l=1}^{j} \int_{N B} P_{j, l}(x) \chi^{(l)}(F(x)) d x d\left(u_{1} \ldots u_{m}\right) \\
& =\sum_{|\alpha|=j} c(\alpha) \int_{T^{m}} \sum_{l=1}^{j} \int_{\mathbb{R}^{s}} P_{j, l}(x) \chi^{(l)}(F(x)) d x d\left(u_{1} \ldots u_{m}\right) .
\end{aligned}
$$

Here we used $\widehat{\chi^{(l)}}(t)=(2 \pi i t)^{l} \widehat{\chi}(t)$ and the fact that $\chi^{(l)}(F(x))=0$ if $x \notin$ $N B$. In the case $j=1$ Lemma 3.1 yields

$$
\begin{aligned}
H_{1} & =-\iint_{T \mathbb{R}^{s}} P_{1,1}(x) \chi^{(1)}(F(x)) d x d u_{1} \\
& =-\int_{\mathbb{R}^{s}} \sum_{i=1}^{s} \frac{\partial F}{\partial x_{i}}(x) \chi^{(1)}(F(x)) d x \int_{T} u_{1}^{(i)} d u_{1}=0 .
\end{aligned}
$$

Remember that $T=(-1 / 2,1 / 2]^{s}$. For $j \geq 1$ we prove that

$$
\sum_{l=1}^{j+1} \int_{\mathbb{R}^{s}} P_{j+1, l}(x) \chi^{(l)}(F(x)) d x=0 .
$$

This implies $H_{j}=0$ for $j \geq 2$. To prove (19) set

$$
H_{j, l}=\int_{\mathbb{R}^{s}} \sum_{i=1}^{s} \frac{\partial}{\partial x_{i}}\left(P_{j, l}(x)\right) u_{j+1}^{(i)} \chi^{(l)}(F(x)) d x .
$$

Using partial integration one obtains, for $2 \leq l \leq j+1$,

$$
\begin{aligned}
\int_{\mathbb{R}^{s}} P_{j, l-1}(x) & \sum_{i=1}^{s} \frac{\partial F}{\partial x_{i}}(x) u_{j+1}^{(i)} \chi^{(l)}(F(x)) d x \\
& =\sum_{i=1}^{s} u_{j+1}^{(i)} \int_{\mathbb{R}^{s}} P_{j, l-1}(x) \frac{\partial}{\partial x_{i}}\left(\chi^{(l-1)}(F(x))\right) d x \\
& =-\sum_{i=1}^{s} u_{j+1}^{(i)} \int_{\mathbb{R}^{s}} \frac{\partial}{\partial x_{i}}\left(P_{j, l-1}(x)\right) \chi^{(l-1)}(F(x)) d x=-H_{j, l-1} .
\end{aligned}
$$


This together with the representation of $P_{j+1, l}$ in Lemma 3.1 implies

$$
\begin{aligned}
\int_{\mathbb{R}^{s}} P_{j+1,1}(x) \chi^{(1)}(F(x)) d x & =H_{j, 1}, \\
\int_{\mathbb{R}^{s}} P_{j+1, l}(x) \chi^{(l)}(F(x)) d x & =H_{j, l}-H_{j, l-1} \quad(2 \leq l \leq j), \\
\int_{\mathbb{R}^{s}} P_{j+1, j+1}(x) \chi^{(j+1)}(F(x)) d x & =-H_{j, j} .
\end{aligned}
$$

Adding these $j+1$ equations yields (19). This completes the proof of Lemma 3.2 and the proof of the Proposition.

4. Proof of Theorem 3. We have to prove that $\Omega(F)>d$ implies (A) $-(\mathrm{D})$ of the Proposition. We start with (D). It is only here that we use, for inhomogeneous $F$, the more sophisticated definition (10) instead of (9).

Lemma 4.1. If $0<\omega<\Omega(F)$ then

$$
\int_{N B^{\prime}} e(t F(u)) d u \ll N^{s} \min \left(1,\left(|t| N^{d}\right)^{-\omega}\right)
$$

uniformly for all boxes $B^{\prime} \subseteq B$ with sides parallel to the coordinate axes.

Proof. The estimate is trivial for $|t| \leq N^{-d}$. If $|t|>N^{-d}$ the substitution $u=Q^{-1} x$ with $Q N \geq 1$ yields

$$
\begin{aligned}
\int_{N B^{\prime}} e(t F(u)) d u & =Q^{-s} \int_{Q N B^{\prime}} e\left(t F\left(Q^{-1} x\right)\right) d x \\
& =Q^{-s}\left(\sum_{n \in Q N B^{\prime} \cap \mathbb{Z}^{s}} e\left(t F\left(Q^{-1} n\right)\right)+O\left(|t| N^{d}(Q N)^{s-1}\right)\right) .
\end{aligned}
$$

To prove (20) cover $Q N B^{\prime}$ by cubes of the form $n+T, T=[-1 / 2,1 / 2)^{s}$. There are at most $O\left((Q N)^{s-1}\right)$ cubes which intersect the boundary of $Q N B^{\prime}$. Furthermore, for $x \in n+T$ with $n \in Q N B^{\prime}$, one finds

$$
e\left(t F\left(Q^{-1} x\right)\right)=e\left(t F\left(Q^{-1} n\right)\right)+O\left(|t| Q^{-1} N^{d-1}\right)
$$

since $\frac{\partial}{\partial x_{i}}\left(t F\left(Q^{-1} x\right)\right) \ll|t| Q^{-1} \frac{\partial F}{\partial x_{i}}\left(Q^{-1} x\right) \ll|t| Q^{-1} N^{d-1}$. This proves (20). The exponential sum in (20) has the form

$$
\sum_{n \in Q N B^{\prime} \cap \mathbb{Z}^{s}} e\left(t Q^{-d} F^{(d)}(n)+P(n)\right)
$$

with a polynomial $P \in \mathbb{R}\left[X_{1}, \ldots, X_{s}\right]$ of degree strictly smaller than $d$. For $0<\Delta \leq 1$ choose $Q$ such that $|t| Q^{-d}=(Q N)^{\Delta-d}$. Then $Q N \geq 1$ and $|t| Q^{-d}$ lies on the boundary of $\mathfrak{M}_{\Delta}(1,1)$. By the definition (10) of $\Omega(F)$ the exponential sum is $\ll(Q N)^{s-\omega \Delta}$. If $F$ is homogeneous the same follows 
from the alternative definition (9). Now (20) implies

$$
\begin{aligned}
\int_{N B^{\prime}} e(t F(u)) d u & \ll Q^{-s}(Q N)^{s-\omega \Delta}+|t| Q^{-1} N^{s+d-1} \\
& \ll N^{s-\omega d}|t|^{-\omega}+N^{s}\left(|t| N^{d}\right)^{1-1 / \Delta} .
\end{aligned}
$$

Both terms on the right hand side are equal if we set $\Delta=(1+\omega)^{-1} \in(0,1]$.

LEMma 4.2. $\Omega(F)>d$ implies that condition $(\mathrm{C})$ of the Proposition is satisfied.

Proof. Condition (C) is trivially satisfied if $|t| \leq N^{-d}$. If $N^{-d}<|t| \leq$ $N^{1-d}$ choose $\Delta(t)$ such that $|t|=N^{\Delta(t)-d}$, i.e. $\Delta(t)=d+\log |t| / \log N$. The condition $N^{-d}<|t| \leq N^{1-d}$ ensures $\Delta(t) \in(0,1]$. With this choice $t$ lies on the boundary of $\mathfrak{M}_{\Delta(t)}(1,1)$. Hence $t \in \mathfrak{m}_{\Delta(t)}$ and the definition (10) or (9) implies, for every $\Omega(F)>\omega>d$,

$$
\sum_{n \in N B^{\prime} \cap \mathbb{Z}^{s}} e(t F(n+u)) \ll N^{s-\omega \Delta(t)} \ll N^{s-\omega d}|t|^{-\omega}
$$

uniformly for all $u \in B$ and all boxes $B^{\prime} \subseteq B$ with sides parallel to the coordinate axes. This proves $(\mathrm{C})$.

To verify conditions (A) and (B) of the Proposition, we split the domain of integration into a part covered by minor arcs and a second part covered by major arcs.

LEMMA 4.3 (minor arcs). If $\Omega(F)>d$ and $0<\Delta<1$ then

$$
\begin{aligned}
\int_{\mathfrak{m}_{\Delta}}\left|S_{N}(t)\right| d t & \ll N^{s-d}, \\
\int_{\left(N^{1-d}, 1\right] \cap \mathfrak{m}_{\Delta}}\left|S_{N}(t)\right| \frac{d t}{t} & \ll N^{s-d} .
\end{aligned}
$$

Proof. We prove (22). The proof of (21) is analogous; see [6, p. 24, Lemma 4.B], for an even sharper estimate. Choose $\omega$ such that $\Omega(F)>$ $\omega>d$. If $\Delta=1$ the definition of $\Omega(F)$ implies $S_{N}(t) \ll N^{s-\omega}$ for all $t \in \mathfrak{m}_{1}$. Hence

$$
\int_{\left(N^{1-d}, 1\right] \cap \mathfrak{m}_{1}}\left|S_{N}(t)\right| \frac{d t}{t} \ll N^{s-\omega} \int_{\left(N^{1-d}, 1\right]} \frac{d t}{t} \ll N^{s-\omega} \log N \ll N^{s-d} .
$$

If $0<\Delta<1$ we split $(\Delta, 1]$ into subintervals $\left(\Delta_{i-1}, \Delta_{i}\right]$, where $\Delta=\Delta_{0}<$ $\Delta_{1}<\ldots<\Delta_{n}=1$. Then

$$
\mathfrak{m}_{\Delta}=\left((\mathbb{R} / \mathbb{Z}) \backslash \mathfrak{M}_{1}\right) \cup \bigcup_{i=1}^{n} \mathfrak{M}_{\Delta_{i}} \backslash \mathfrak{M}_{\Delta_{i-1}}=\mathfrak{m}_{1} \cup \bigcup_{i=1}^{n} \mathfrak{r}_{i}
$$


where $\mathfrak{r}_{i}=\mathfrak{M}_{\Delta_{i}} \backslash \mathfrak{M}_{\Delta_{i-1}} \subseteq \mathfrak{M}_{\Delta_{i}}$. Since $\mathfrak{M}_{\Delta}$ has Lebesgue measure

$$
\lambda\left(\mathfrak{M}_{\Delta}\right) \ll \sum_{1 \leq a \leq q \leq N^{\Delta}} q^{-1} N^{\Delta-d} \ll N^{2 \Delta-d},
$$

it follows that $\lambda\left(\mathfrak{r}_{i}\right) \ll N^{2 \Delta_{i}-d}$. Furthermore, the definition of $\Omega(F)$ yields for $t \in \mathfrak{r}_{i} \subseteq \mathfrak{m}_{\Delta_{i-1}}$ the estimate $S_{N}(t) \ll N^{s-\omega \Delta_{i-1}}$. Hence we obtain

$$
\begin{aligned}
\int_{\left(N^{d-1}, 1\right] \cap \mathfrak{m}_{\Delta}}\left|S_{N}(t)\right| \frac{d t}{t} & \ll \int_{\left(N^{d-1}, 1\right] \cap \mathfrak{m}_{1}}\left|S_{N}(t)\right| \frac{d t}{t}+\sum_{i=1}^{n} \int_{\left(N^{d-1}, 1\right] \cap \mathfrak{r}_{i}}\left|S_{N}(t)\right| \frac{d t}{t} \\
& \ll N^{s-d}+\sum_{i=1}^{n} N^{s-\omega \Delta_{i-1}} \int_{\left(N^{d-1}, 1\right] \cap \mathfrak{r}_{i}} \frac{d t}{t} .
\end{aligned}
$$

Since $\mathfrak{r}_{i} \subseteq \mathfrak{M}_{\Delta_{i}}$ we consider (for $(a, q) \neq(1,1)$ )

$$
\int_{\mathfrak{M}_{\Delta}(q, a) \cap(0,1]} \frac{d t}{t}=\int_{\frac{a}{q}-\frac{1}{q} N^{\Delta-d}}^{\frac{a}{q}+\frac{1}{q} N^{\Delta-d}} \frac{d t}{t}=\log \frac{1+\frac{1}{a} N^{\Delta-d}}{1-\frac{1}{a} N^{\Delta-d}} \ll \frac{1}{a} N^{\Delta-d} .
$$

It follows that

$$
\int_{\left(N^{1-d}, 1\right] \cap \mathfrak{M}_{\Delta}} \frac{d t}{t} \ll \sum_{1 \leq a \leq q \leq N^{\Delta}} \frac{1}{a} N^{\Delta-d} \ll N^{\Delta-d} \sum_{1<q \leq N^{\Delta}} \log q \ll N^{2 \Delta-d} \log N .
$$

Altogether we obtain

$$
\begin{aligned}
\int_{\left(N^{d-1}, 1\right] \cap \mathfrak{m}_{\Delta}}\left|S_{N}(t)\right| \frac{d t}{t} & \ll N^{s-d}+\sum_{i=1}^{n} N^{s-d-(\omega-2) \Delta_{i-1}+2\left(\Delta_{i}-\Delta_{i-1}\right)} \log N \\
& \ll N^{s-d}+N^{s-d-(\omega-2) \Delta+2 \varepsilon} \ll N^{s-d},
\end{aligned}
$$

if we choose $\Delta_{i}-\Delta_{i-1}<\varepsilon$ sufficiently small. This proves (22) for every $\Delta \in(0,1]$.

Lemma 4.4 (major arcs). If $\Omega(F)>2$ and $0<\Delta<1 / 4$ then

$$
\begin{aligned}
\int_{\mathfrak{M}_{\Delta}}\left|S_{N}(t)\right| d t & \ll N^{s-d}, \\
\int_{\left(N^{1-d}, 1\right] \cap \mathfrak{M}_{\Delta}}\left|S_{N}(t)\right| \frac{d t}{t} & \ll N^{s-d} .
\end{aligned}
$$

Proof. If $F$ is a polynomial with integer coefficients and $t$ is close to a rational number with small denominator, then $S_{N}(t)$ can be evaluated asymptotically. It is well known (cf. [6, p. 26, Lemma 5.A]) that for every $t \in \mathfrak{M}_{\Delta}(q, a)$, we have

$$
S_{N}(t)=S\left(\frac{a}{q}\right) G_{0}\left(t-\frac{a}{q}\right)+O\left(q N^{s-1+\Delta}\right),
$$


where

$$
S\left(\frac{a}{q}\right)=q^{-s} \sum_{n \in q(0,1]^{s} \cap \mathbb{Z}^{s}} e\left(\frac{a}{q} F(n)\right), \quad G_{0}(t)=\int_{N B} e(t F(u)) d u .
$$

Since $a / q$ with $(a, q)=1$ lies in $\mathfrak{M}_{1}(q, a)$ with $N=q$, the definition of $\Omega(F)$ implies

$$
S\left(\frac{a}{q}\right) \ll q^{-\omega}
$$

for every $\omega<\Omega(F)$. Additionally, by Lemma 4.1, $G_{0}(t) \ll N^{s} \min \left(1,\left|t N^{d}\right|^{-\omega}\right)$ for $\omega<\Omega(F)$. Since $\Omega(F)>2$ we can choose $\omega>2$. Using these estimates it is easy to prove (24) and (25). We demonstrate (25). Since

$$
\left|t-\frac{a}{q}\right| \leq \frac{1}{q} N^{\Delta-d} \quad \text { for } t \in \mathfrak{M}_{\Delta}(q, a),
$$

it follows that $t \geq a /(2 q)$. Hence

$$
\begin{aligned}
\int_{\mathfrak{M}_{\Delta}(q, a) \cap(0,1]}\left|S_{N}(t)\right| \frac{d t}{t} \\
\ll\left|S\left(\frac{a}{q}\right)\right| \frac{q}{a} \int_{|u| \leq \frac{1}{q} N^{\Delta-d}}\left|G_{0}(u)\right| d u+q N^{s-1+\Delta} \int_{\mathfrak{M}_{\Delta}(q, a) \cap(0,1]} \frac{d t}{t} .
\end{aligned}
$$

The substitution $u=N^{-d} v$ yields

$$
\begin{aligned}
\int_{|u| \leq \frac{1}{q} N^{\Delta-d}}\left|G_{0}(u)\right| d u & =N^{-d} \int_{|v| \leq \frac{1}{q} N^{\Delta}}\left|G_{0}\left(N^{-d} v\right)\right| d v \\
& \ll N^{s-d} \int_{|v| \leq \frac{1}{q} N^{\Delta}} \min \left(1,|v|^{-\omega}\right) d v \ll N^{s-d} .
\end{aligned}
$$

Together with (23) and (27) we obtain

$$
\begin{aligned}
\int_{\left(N^{1-d}, 1\right] \cap \mathfrak{M}_{\Delta}}\left|S_{N}(t)\right| \frac{d t}{t} & \ll N^{s-d} \sum_{1 \leq a \leq q \leq N^{\Delta}}\left(a^{-1} q^{1-\omega}+a^{-1} q N^{2 \Delta-1}\right) \\
& \ll N^{s-d}\left(1+N^{4 \Delta-1}\right) \log N \ll N^{s-d} .
\end{aligned}
$$

5. Proof of Theorem 2. Let $F_{0}(X)=\sum_{i=1}^{s} \lambda_{i} X_{i}^{d}$ with integer coefficients $\lambda_{i}>0$. It is known that $\Omega\left(F_{0}\right) \geq s 2^{1-d}$ (see $[6$, p. 24] and the remarks following (11)). Hence Theorem 3 implies $P_{F_{0}}(R) \ll R^{s / d-1}$ if $s>d 2^{d-1}$. For large $d$ this can be substantially improved by Vinogradov's mean value theorem. We prove that $(\mathrm{A})-(\mathrm{D})$ of the Proposition are satisfied if $s>\varrho_{0}(d)$, where $\varrho_{0}(d)$ is an explicitly computable function which satisfies $\varrho_{0}(d) \sim 2 d^{3} \log d$ for $d \rightarrow \infty$. 
First we prove that (C) and (D) are satisfied if $s>d^{2}, d>2$. To do this we establish (7) and (8) with $\omega=s / d$. By [2, Theorem 2.2] (the second derivative test), it follows that

$$
\sum_{M<n \leq M^{\prime}} e\left(t(n+u)^{d}\right) \ll\left(|t| M^{d-2}\right)^{-1 / 2}+M\left(|t| M^{d-2}\right)^{1 / 2}
$$

uniformly for $u \in[-1,1]$ and $1 \leq M<M^{\prime} \leq 2 M$. Splitting $[0, N]$ into dyadic intervals of the form $\left(2^{j-1} \bar{U}, 2^{j} U\right]$ with $\bar{U}=|t|^{-1 / d}$ we obtain

$$
\begin{aligned}
\sum_{0 \leq n \leq N} e\left(t(n+u)^{d}\right) & \ll 1+U+\sum_{j}\left(|t|^{-1 / 2}\left(2^{j} U\right)^{1-d / 2}+|t|^{1 / 2}\left(2^{j} U\right)^{d / 2}\right) \\
& \ll 1+U+|t|^{-1 / 2} U^{1-d / 2}+|t|^{1 / 2} N^{d / 2} \\
& \ll|t|^{-1 / d}+|t|^{1 / 2} N^{d / 2} .
\end{aligned}
$$

It follows that

$$
\sum_{n \in N B^{\prime}} e\left(t F_{0}(n+u)\right) \ll\left(|t|^{-1 / d}+|t|^{1 / 2} N^{d / 2}\right)^{s} \ll|t|^{-s / d}
$$

if $|t| \leq N^{1-d}$. This proves (7) with $\omega=s / d$. To prove (D) observe that for $t>0$

$$
\int_{0}^{N} e\left(t x^{d}\right) d x=t^{-1 / d} d^{-1} \int_{0}^{t N^{d}} \xi^{1 / d-1} e(\xi) d \xi \ll t^{-1 / d}
$$

(the last integral is bounded by an absolute constant). This proves (8) with $\omega=s / d$.

Next we prove (A) and (B). Let

$$
f(t)=\sum_{1 \leq n \leq N} e\left(t n^{d}\right),
$$

then $S_{N}(t)=\prod_{i=1}^{s}\left(1+2 f\left(\lambda_{i} t\right)\right)$. By Hölder's inequality it is sufficient to prove

$$
\int_{(0,1]}|f(t)|^{s} d t \ll N^{s-d} \text { and } \int_{\left(\lambda_{i} N^{1-d}, 1\right]}|f(t)|^{s} \frac{d t}{t} \ll N^{s-d} .
$$

To estimate the special function $f(t)$ one can work with larger major arcs. Let $N=\left\lceil(R+1)^{1 / d}\right\rceil+1 / 2$ and set

$$
\mathfrak{M}(q, a)=\left\{t \in \mathbb{R} / \mathbb{Z}|| t-\frac{a}{q} \mid \leq \frac{P}{q R}\right\}, \quad P=\frac{N}{2 d} .
$$

Write $\mathfrak{M}$ for the union of the $\mathfrak{M}(q, a)$ with $1 \leq a \leq q \leq P$ and $(a, q)=1$, and set $\mathfrak{m}=(\mathbb{R} / \mathbb{Z}) \backslash \mathfrak{M}$.

Lemma 5.1 (major arcs). If $s>2 d$ and $c>0$ then

$$
\int_{\mathfrak{M}}|f(t)|^{s} d t \ll N^{s-d} \quad \text { and } \int_{\left(c N^{1-d}, 1\right] \cap \mathfrak{M}}|f(t)|^{s} \frac{d t}{t} \ll N^{s-d} .
$$


Proof. By [9, Theorem 4.1], for $t \in \mathfrak{M}(q, a)$ and any $\varepsilon>0$,

$$
f(t)=\frac{1}{q} S\left(\frac{a}{q}\right) v\left(t-\frac{a}{q}\right)+O\left(q^{1 / 2+\varepsilon}\right),
$$

where, by [9, Theorem 4.2 and Lemma 2.8],

$$
\frac{1}{q} S\left(\frac{a}{q}\right) \ll q^{-1 / d} \text { and } \quad v(t) \ll \min \left(N,|t|^{-1 / d}\right) .
$$

This yields

$$
\int_{\left(c N^{1-d}, 1\right] \cap \mathfrak{M}}|f(t)|^{s} \frac{d t}{t} \ll \sum_{1 \leq a \leq q \leq P}\left(q^{-s / d} \int_{|u| \leq P /(q R)}|v(u)|^{s} d u+q^{s / 2+\varepsilon} \frac{P}{q R}\right) \frac{q}{a} .
$$

Since

$$
\int_{|u| \leq P /(q R)}|v(u)|^{s} d u \ll N^{s-d}+\int_{\left(N^{-d}, P /(q R)\right]} u^{-s / d} d u \ll N^{s-d},
$$

we obtain, for $s>2 d$,

$$
\int_{\left(c N^{1-d}, 1\right] \cap \mathfrak{M}}|f(t)|^{s} \frac{d t}{t} \ll N^{s-d} \sum_{q \leq N} q^{1-s / d} \log q+N^{1-d} \sum_{q \leq N} q^{s / 2+2 \varepsilon} \ll N^{s-d} .
$$

This proves the second assertion of the lemma. The first one follows in the same way.

Finally, we estimate the contribution of the minor arcs to (28). Since

$$
\int_{\left(\lambda_{i} N^{d-1}, 1\right] \cap \mathfrak{m}}|f(t)|^{s} \frac{d t}{t} \ll N^{1-d} \int_{\mathfrak{m}}|f(t)|^{s} d t
$$

(28) is a consequence of Lemma 5.1 and the following lemma.

LEMMA 5.2 (minor arcs). There is an explicitly computable function $\varrho_{0}(d)$, which satisfies $\varrho_{0}(d) \sim 2 d^{3} \log d$ for $d \rightarrow \infty$, such that for $s \geq \varrho_{0}(d)$,

$$
\int_{\mathfrak{m}}|f(t)|^{s} d t \ll N^{s-2 d+1} .
$$

Proof. We use Wooley's refinement of Vinogradov's mean value theorem. The original form of the mean value theorem yields Lemma 5.2 with $\varrho_{0}(d) \sim$ $4 d^{3} \log d$. By [9, Theorem 5.6], there is an explicitly computable function $\sigma(d)$ such that for $t \in \mathfrak{m}$,

$$
f(t) \ll N^{1-\sigma(d)} \log N .
$$

We have $\sigma(d) \sim\left(2 d^{2} \log d\right)^{-1}$ for $d \rightarrow \infty$. Furthermore, by [9, Theorem 5.5 and (5.37)], for every integer $l \geq 1$,

$$
\int_{(0,1]}|f(t)|^{2 d l} d t \ll N^{2 d l-d+\eta_{l}(d)},
$$


where

$$
\eta_{l}(d)=\frac{1}{2} d(d-1)\left(1-\frac{5}{4 d}\right)^{l-1} .
$$

These estimates imply, for every $l \geq 1$,

$$
\begin{aligned}
\int_{\mathfrak{m}}|f(t)|^{s} d t & \left.\ll \sup _{t \in \mathfrak{m}}|f(t)|^{s-2 d l}\right) \int_{(0,1]}|f(t)|^{2 d l} d t \\
& \ll N^{(s-2 d l)(1-\sigma(d))+2 d l-d+\eta_{l}(d)}(\log N)^{s-2 d l} .
\end{aligned}
$$

There is an $l$ such that the right hand side is $\ll N^{s-2 d+1}$ if

$$
s>\min _{l}\left\{\frac{\eta_{l}(d)}{\sigma(d)}+2 d l\right\}+\frac{d-1}{\sigma(d)}=\varrho_{0}(d),
$$

say. By [9, Theorem 5.7], the minimum is $\ll d^{2} \log d$, thus $\varrho_{0}(d) \sim 2 d^{3} \log d$ for $d \rightarrow \infty$.

We remark that for small $d$ Theorem 2 can be further sharpened. For instance, Hua's lemma $\left(\left[9\right.\right.$, Lemma 2.5]) can be used to prove $P_{F_{0}}(R) \ll$ $R^{s / d-1}$ for $s>2^{d+1}-2$.

\section{References}

[1] V. Bentkus and F. Götze, Lattice points in multidimensional bodies, Forum Math. 13 (2001), 149-225.

[2] S. W. Graham and G. Kolesnik, Van der Corput's Method of Exponential Sums, London Math. Soc. Lecture Note Ser. 126, Cambridge Univ. Press, 1991.

[3] E. Krätzel, Lattice Points, Kluwer, Dordrecht, 1988.

[4] E. Landau, Über Gitterpunkte in mehrdimensionalen Ellipsoiden, Math. Z. 21 (1924), 126-132.

[5] B. Randol, A lattice point problem I, II, Trans. Amer. Math. Soc. 121, 125 (1966), 257-268, 101-113.

[6] W. M. Schmidt, Analytische Methoden für Diophantische Gleichungen, DMV Sem. 5, Birkhäuser, 1984.

[7] —, Bounds for exponential sums, Acta Arith. 44 (1984), 281-297.

[8] —, The density of integer points on homogeneous varieties, Acta Math. 154 (1985), 243-296.

[9] R. C. Vaughan, The Hardy-Littlewood Method, 2nd ed., Cambridge Tracts in Math. 125, Cambridge Univ. Press, 1997.

[10] A. Walfisz, Über Gitterpunkte in mehrdimensionalen Ellipsoiden, Math. Z. 19 (1924), 300-307.

Institut für Statistik

Technische Universität Graz

A-8010 Graz, Austria

E-mail: mueller@stat.tu-graz.ac.at 